\title{
HIV Disease Progression in Seroconvertors from the CAPRISA 004 Tenofovir Gel Pre-exposure Prophylaxis Trial
}

\author{
Nigel J. Garrett, MD, MSc, * Lise Werner, MSc, * Nivashnee Naicker, MD, * Vivek Naranbhai, MD, PhD, *† \\ Sengeziwe Sibeko, MD, ${ }^{*} \neq$ Natasha Samsunder, BSc, ${ }^{*}$ Clive Gray, PhD, ${ }^{*} \xi \|$ Carolyn Williamson, PhD, ${ }^{*} \xi \|$ \\ Lynn Morris, PhD, *\|ף Quarraisha Abdool-Karim, PhD,*\# and Salim S. Abdool-Karim, MD, PhD*\#
}

\begin{abstract}
Background: Although antiretroviral pre-exposure prophylaxis prevents HIV acquisition, it is not known if it alters HIV disease progression. This study assesses whether tenofovir gel impacted on disease progression among CAPRISA 004 microbicide trial seroconvertors.
\end{abstract}

Received for publication May 25, 2014; accepted August 29, 2014.

From the *Centre for the AIDS Programme of Research in South Africa (CAPRISA), University of KwaZulu-Natal, Durban, South Africa; $†$ Wellcome Trust Centre for Human Genetics, University of Oxford, Oxford, United Kingdom; $\$$ Nuffield Department of Medicine, University of Oxford, Oxford, United Kingdom; §Institute of Infectious Diseases and Molecular Medicine, University of Cape Town, Cape Town, South Africa; |National Health Laboratory Service, South Africa; IAIDS Virus Research Unit, National Institute for Communicable Diseases, Johannesburg, South Africa; and \#Department of Epidemiology, Columbia University, New York, NY USA.

Supported by the National Institute of Allergy and Infectious Disease, National Institutes of Health (NIH) (Grant AI51794), the National Research Foundation (Grant 67385), the Columbia University-Southern African Fogarty AIDS International Training and Research Program (AITRP) funded by the Fogarty International Center, NIH (Grant D43TW00231) and a training grant from LifeLab, a biotechnology centre of the South African Government Department of Science and Technology. The parent trial (CAPRISA 004) was supported by the United States Agency for International Development (USAID), FHI360 [USAID cooperative agreement \# GPO-A-00-05-00,022-00, contract \# 132119], and the Technology Innovation Agency (LIFElab). Tenofovir was provided by Gilead Sciences and the gel was manufactured and supplied for the CAPRISA 004 trial by CONRAD. The current studies are part of the CAPRISA TRAPS (Tenofovir gel Research for AIDS Prevention Science) Program, which is funded by CONRAD, Eastern Virginia Medical School [USAID co-operative Grant GP00-08-00005-00, subproject agreement \# PPA-09-046]. The views expressed by the authors do not necessarily reflect the views of USAID, Gilead Sciences, Eastern Virginia Medical School or CONRAD.

Preliminary data were presented at the Conference on Retroviruses and Opportunistic Infections 2014, March 2014, Boston, MA.

S.S.A.K. and Q.A.K. are co-inventors of 2 pending patents $(61 / 354.050$ and 61/357.892) of tenofovir gel against HSV-1 and HSV-2 with scientists from Gilead Sciences. The remaining authors have no funding or conflicts of interest to disclose.

N.J.G., L.W., N.N., V.N., S.S., N.S., C.G., C.W., L.M., Q.A.K., and S.S.A.K. contributed to study design, protocol writing, data collection, analysis and interpretation of data. N.J.G. drafted the manuscript, whereas all other authors reviewed the final version of the manuscript.

Supplemental digital content is available for this article. Direct URL citations appear in the printed text and are provided in the HTML and PDF versions of this article on the journal's Web site (www.jaids.com).

Correspondence to: Nigel J. Garrett, MD, MSc, Centre for the AIDS Programme of Research in South Africa (CAPRISA), 2nd Floor, Doris Duke Medical Research Institute, Nelson R Mandela School of Medicine, University of KwaZulu-Natal, 719 Umbilo Road (Private Bag X7), Congella, 4013 Durban, South Africa (e-mail: nigel.garrett@caprisa.org).

Copyright $(C 2014$ by Lippincott Williams \& Wilkins
Methods: Eighty-three seroconvertors from the tenofovir and placebo gel arms of the CAPRISA 004 trial were monitored prospectively for a minimum of 2 years by CD4 count and viral load (VL). Linear mixed models were fitted to HIV VL, and log rank test was used to compare time to reach CD4 counts of $<350$ cells per microliter.

Results: Median 2-week postinfection VL was 4.74 and $4.45 \log$ copies per milliliter in women assigned to tenofovir gel $(\mathrm{n}=32)$ and placebo gel $(\mathrm{n}=51)(P=0.189)$. Corresponding 12-month postinfection VLs were 4.24 and $3.70 \log$ copies per milliliter $(P=$ 0.016). After adjusting for clinical and behavioral characteristics and protective HLA alleles, mean VLs within the first 2 years were 4.51 and $4.02 \log$ copies per milliliter in women from the tenofovir and placebo arms $(P=0.013)$. Among women with vaginal tenofovir measurements, mean VLs were 4.53 and $4.60 \mathrm{log}$ copies per milliliter in those with detectable versus undetectable levels $(P=0.840)$. Overall mean CD4 counts were 463 and 514 cells per microliter in women assigned to tenofovir and placebo $(P=0.290)$. Thirty-two women $(38.6 \%)$ reached CD4 counts of $<350$ cells per microliter at median 9.4 months postinfection, $13(40.6 \%)$ from the tenofovir and $19(37.3 \%)$ from the placebo arms $(P=0.786)$.

Conclusions: Tenofovir gel had no impact on postinfection CD4 counts or the rate of CD4 decline. Although seroconvertors from the tenofovir arm experienced higher VLs, this did not result in a need for earlier antiretroviral therapy.

Key Words: pre-exposure prophylaxis, HIV acquistion, HIV disease progression, viral load, tenofovir gel

(J Acquir Immune Defic Syndr 2015;68:55-61)

\section{INTRODUCTION}

Randomized controlled clinical trials have demonstrated that systemic pre-exposure prophylaxis (PrEP) prevents HIV acquisition in men who have sex with men, ${ }^{1}$ intravenous drug users, ${ }^{2}$ and heterosexual men and women., ${ }^{3,4}$ This has led to the licensing of oral tenofovir/emtricitabine (Truvada) as PrEP in HIV-uninfected individuals at risk of HIV infection. In addition, the use of antiretrovirals in topical formulations to prevent HIV acquisition has been actively pursued, but with variable results, ${ }^{5,6}$ possibly attributable to differences in adherence between trials. Although concerns about drug side effects in healthy individuals, ${ }^{7,8}$ selection for drug resistance, and risk compensation have largely been allayed by recent studies, ${ }^{9-11}$ the effect of failed PrEP on disease course remains an area of 
concern. Animal studies have suggested that macaques, which acquired Simian/Human Immunodeficiency Virus after failed PrEP, may have lower acute viremia and be at higher risk for the acquisition of drug-resistant virus. ${ }^{12,13}$

Few studies have assessed the effect of PrEP use on markers of HIV disease progression in individuals who acquire HIV despite PrEP. ${ }^{14}$ In the CAPRISA 004 1\% tenofovir gel microbicide trial, ${ }^{5}$ women who acquired HIV in the tenofovir and placebo arms were offered enrollment into the CAPRISA 002 Acute Infection cohort study. ${ }^{15}$ Research on these women from acute HIV infection has already established that the diversity of the transmitting virus was similar between seroconvertors from the $2 \mathrm{arms}^{16}$ and tenofovir gel did not seem to select for viruses with enhanced fitness. ${ }^{17}$ Interestingly, T-cell responses were relatively preserved during early infection among women who were randomized to receive tenofovir gel but who seroconverted. ${ }^{18}$ In addition, sensitive drug resistance screening of plasma and cervicovaginal lavage specimen collected during acute HIV infection did not reveal any PrEP-related tenofovir drug resistance mutations. ${ }^{19}$

Despite these reassuring findings, data from long-term follow-up of participants who seroconverted after tenofovir gel exposure are required to address the safety of topical PrEP and to alleviate remaining concerns about its implementation. The CAPRISA 004 trial and the already established CAPRISA 002 Acute Infection study provided us with the opportunity to offer PrEP trial seroconvertors long-term follow-up. Therefore, the objective of this study was to compare markers of HIV disease progression among women who seroconverted during the CAPRISA 004 trial and were exposed to either 1\% vaginal tenofovir gel or placebo.

\section{METHODS}

\section{Design and Study Population}

Women who acquired HIV infection during the CAPRISA 004 study, a randomized controlled, doubleblinded trial to assess the safety and effectiveness of the vaginal microbicide $1 \%$ tenofovir gel, were offered enrollment into the CAPRISA 002 Acute Infection cohort study. Recruitment methods and eligibility criteria for both studies were previously described in detail. ${ }^{5,15}$

Briefly, 889 sexually active, HIV-uninfected women aged 18-40 years from urban and rural settings in South Africa participated in the CAPRISA 004 study between May 2007 and March 2010. At each monthly visit, women tested for HIV with 2 rapid point-of-care antibody tests. Suspected seroconvertors underwent HIV ribonucleic acid (RNA) polymerase chain reaction (PCR) and laboratory enzyme-linked immunosorbent assay testing to confirm the HIV diagnosis. Product hold was instituted upon confirmation of HIV acquisition. The date of infection was estimated as the midpoint between the last negative and first positive antibody test, or if a woman had tested negative on rapid antibody tests but positive on retrospective PCR testing, the infection date was estimated to be 14 days before the positive PCR result.

A total of 98 women acquired HIV during the trial, 38 in the tenofovir and 60 in the placebo arm. Of these, 83 women consented to enroll into the CAPRISA 002 study. Participants were assessed at enrollment and were then followed-up fortnightly until 3 months, monthly during early infection until 12 months, and 3-monthly, or if medically indicated, thereafter, until antiretroviral therapy (ART) initiation. Participants had at least 2 years of follow-up. ART was commenced at a CD4 count of $<350$ cells per microliter or an AIDS-defining diagnosis, as per South African Adult Treatment Guidelines. ${ }^{20}$

Demographic and behavioral data were collected in both studies. Information on sexual behavior, condom, and contraceptive use and adherence to the 1\% tenofovir and placebo gels was collected in CAPRISA 004 and was previously reported. ${ }^{11}$

\section{Laboratory Methods}

During the CAPRISA 004 trial, 2 HIV rapid tests, Determine HIV 1/2 (Abbott Laboratories, Chicago, IL) and Uni-Gold Recombigen HIV test (Trinity Biotech, Wicklow, Ireland), were performed at each study visit. Participants with concordantly positive, discordant, or indeterminate results were assessed for possible seroconversion by 2 separate RNA PCR assays (Roche Cobas Amplicor HIV-1 Monitor v1.5; Roche Diagnostics, Branchburg, NJ), 1 week apart.

Once diagnosed and enrolled, participants had HIV viral load (VL) and CD4 ${ }^{+}$T-cell counts using the TruCOUNT method (BD Biosciences, San Jose, CA) performed at each visit. During the course of the study, the VL PCR assay switched from Roche Ampliprep-Amplicor to Roche Taqman version 1.0 on June 1, 2010, and then to Roche Taqman version 2.0 on January 9, 2012. All analyses were therefore adjusted for assay type, and all samples at the critical 12month postinfection time point were retested on the Taqman version 2.0 assay to eliminate potential measurement bias. The CAPRISA laboratory is accredited by the South African National Accreditation System (ISO15189).

In addition, all women underwent a genital screen for sexually transmitted infections (STIs), including herpes simplex virus (HSV), Chlamydia trachomatis, Neisseria gonorrhoeae, Mycoplasma genitalium, Trichomonas vaginalis PCR and hepatitis B core antibody testing, at enrollment and 6-monthly during the study. HLA typing at 4-digit resolution was performed using sequence-based typing (Atria AlleleSEQR kits; Abbott, Chicago, IL). Additional laboratory and tenofovir drug level data were available from the CAPRISA 004 trial.

Plasma samples were tested for antiretroviral drug levels in seroconvertors with a VL $<400$ copies per milliliter at 12month postinfection. A liquid chromatography-mass spectrometry (LC-MS/MS) semiquantitative method was used for the detection of efavirenz, nevirapine, lopinavir, and ritonavir developed in-house in the Division of Clinical Pharmacology, University of Cape Town, South Africa. Twenty microliters of plasma was extracted by protein precipitation and analyzed on an AB Sciex API 4000 mass spectrometer (AB Sciex, Framingham, MA). Control samples spiked to the cut-off limits were analyzed with each batch and used to determine the presence or absence of the analytes. A deuterated internal standard was used to monitor the extraction efficiency of the method. 


\section{Outcome Measures}

We defined outcomes as follows: (1) a difference in overall VL measurements and, specifically, at 12 months postinfection, (2) a difference in overall CD4 count measurements and at 12 months postinfection, and (3) the rate of disease progression to CD4 count $<350$ cells per microliter, that is, the need for ART initiation. Decline of a CD4 count to $<350$ cells per microliter was deemed an endpoint, if it occurred at least 6 months postinfection to allow for $\mathrm{CD}^{+}$ T-cell lymphopenia during primary infection that reverted by 6 months.

\section{Statistical Analysis}

Basic descriptive statistics were used to summarize characteristics of seroconvertors at enrollment. Baseline differences between women from the tenofovir and placebo arms were compared using Wilcoxon rank sums and Fisher exact tests.

CD4 count and HIV VL measurements in the first 2 years of infection, at 3-monthly intervals, were described using medians and interquartile ranges. The Wilcoxon rank sum test was used to compare tenofovir and placebo arm seroconvertors at the specific time points. Linear mixed models, accounting for repeated measures, were fitted to all CD4 count and HIV VL measurements in the first 2 years of infection to assess the impact of CAPRISA 004 arm. Unadjusted and adjusted analyses were performed controlling for possible confounders.

Time to CD4 count $<350$ cells per microliter was calculated as the period from estimated date of infection to the date of the first measured CD4 count $<350$ cells per microliter beyond 6 months postinfection. Those who did not reach this low CD4 count were censored at their last postinfection visit. A Kaplan-Meier survival curve and log rank test was used to compare the differences between the arms.

Linear mixed models were used to assess the association between tenofovir concentrations, as measured in the genital tract in the CAPRISA 004 study, and VL in the first 2 years of HIV infection.

Statistical analysis was performed using SAS version 9.3 (SAS Institute Inc., Cary, NC), whereas graphs were prepared using GraphPad Prism 5.

\section{Ethical Considerations}

Ethical approval for the CAPRISA 002 study was obtained from the University of KwaZulu-Natal (E013/04), the University of Cape Town (025/2004), and the University of the Witwatersrand (M040202).

\section{RESULTS}

\section{Cohort Characteristics by Trial Arm}

A total of 83 women of 98 CAPRISA 004 seroconvertors $(84.7 \%)$ consented to participate in the CAPRISA 002 Acute Infection study and have so far contributed 296.1 women-years of ART-naive follow-up with a retention rate of
94.0\% and median follow-up time of 48 months (IQR: $31-$ 57). Recruitment from the 2 trial arms was evenly balanced with $32(84.2 \%)$ of 38 women from the tenofovir gel PrEP arm and $51(85.0 \%)$ of 60 women from the placebo arm. Two thirds of women $(67.5 \%)$ were diagnosed at the rural and one third $(32.5 \%)$ at the urban CAPRISA research sites. The 15 women who did not enroll into the study had similar baseline characteristics to those who did enroll, apart from reporting a lower condom use at their last sex act $(58.3 \%$ vs $84.2 \%, P=$ 0.049 ), and showing lower rates of STI symptoms before seroconversion $(0.0 \%$ vs $32.5 \%, P=0.005)$.

At enrollment into the study, the median age was 22 years (IQR: $21-25)$, most women (81.9\%) had had 1 sexual partner in the previous month with a median of 4 sex-acts per month (IQR: 2-7), and 61.5\% were using depot medroxyprogesterone acetate injections as their main method of contraception (Table 1).

The prevalence of STIs $(40.7 \%)$ and bacterial vaginosis $(55.6 \%)$ was high at enrollment. Specifically, $16.1 \%$ of women were diagnosed with $C$. trachomatis, $6.2 \%$ with $N$. gonorrhoeae, $8.6 \%$ with M. genitalium, and $4.9 \%$ with T. vaginalis. In addition, $11.1 \%$ of women tested positive for HSV by genital fluid PCR.

The median first HIV VL at an estimated median of 2 weeks postinfection was $4.60 \mathrm{log}$ copies per milliliter (IQR: 3.74-5.25) with median of $4.74 \mathrm{log}$ copies per milliliter (IQR: 3.97-5.37) in seroconvertors from the tenofovir arm versus 4.45 log copies per milliliter (IQR: 3.56-5.17) from the placebo arm $(P=0.189)$. The median first CD4 count at an estimated median of 5 weeks (IQR: $3-9$ ) postinfection was 498 cells per microliter (IQR: 393-643) with 467 cells per microliter (IQR: 380-530) in the tenofovir arm versus 519 cells per microliter (IQR: 414-689) in the placebo arm $(P=0.106)$.

Comparing seroconvertors from each trial arm, no statistically significant differences in baseline characteristics were found (Table 1).

\section{Seroconvertors Assigned to Tenofovir Gel Have Higher VLs, but Similar CD4+ ${ }^{+}$-cell Counts, Compared to Placebo Recipients During the First Two Years of Infection}

VL measurements remained higher from acute infection to 24 months postinfection in seroconvertors from the tenofovir compared with the placebo arm (Fig. 1). The estimated overall mean VL for the tenofovir and placebo arms were $4.51 \mathrm{log}$ copies per milliliter $(\mathrm{SE}=0.20)$ and $4.02 \mathrm{log}$ copies per milliliter $(\mathrm{SE}=0.19)$, respectively, during the course of follow-up $(P=0.013)$. The difference between arms was statistically significant by 12 months postinfection, with a median VL of $4.24 \mathrm{log}$ copies per milliliter (IQR: $3.74-$ 4.77) versus 3.70 log copies per milliliter (IQR: 2.60-4.66) in women from the tenofovir and placebo arms, respectively $(P=0.016)$ (Table 1). Although, seroconvertors from the tenofovir arm generally had lower CD4 counts, this difference did not reach statistical significance at any point during the 24-month follow-up (Table 2). Even after adjusting for potential confounders including protective HLA types (B*1302, 
TABLE 1. Baseline Characteristics of CAPRISA 004 Seroconvertors

\begin{tabular}{|c|c|c|c|c|}
\hline Characteristic & All $(\mathbf{n}=\mathbf{8 3})$ & Tenofovir $\operatorname{arm}(n=32)$ & Placebo arm $(n=51)$ & $P^{*}$ \\
\hline Median age at seroconversion in years (IQR) & $22(21-25)$ & $23(20-27)$ & $22(21-25)$ & 0.460 \\
\hline $\begin{array}{l}\text { Median estimated days post infection at CAP002 } \\
\text { enrollment (IQR) }\end{array}$ & $37(24-65)$ & $40(29-83)$ & $36(22-59)$ & 0.133 \\
\hline \multicolumn{5}{|l|}{ \% Sexual partners before seroconversion (n) } \\
\hline More than 1 & $1.2 \%(1)$ & $0.0 \%(0)$ & $2.0 \%(1)$ & 0.690 \\
\hline 1 only & $81.9 \%(68)$ & $78.1 \%(25)$ & $84.3 \%(43)$ & - \\
\hline $\begin{array}{l}\text { Median number of returned used gels at visit before } \\
\text { seroconversion (IQR) }\end{array}$ & $6(3-10)$ & $5(2-10)$ & $8(4-10)$ & 0.287 \\
\hline Median adherence in 004 (IQR) & $59.2 \%(50 \%-100 \%)$ & $56.7 \%(50 \%-100 \%)$ & $60.0 \%(50 \%-100 \%)$ & 0.927 \\
\hline $\begin{array}{l}\% \text { with condom use at last sex reported at visit before } \\
\text { seroconversion }(\mathrm{n})\end{array}$ & $84.2 \%(69)$ & $81.3 \%(26)$ & $86.0 \%(43)$ & 0.758 \\
\hline \% Baseline positive (n) & $56.6 \%(47)$ & $53.1 \%(17)$ & $58.8 \%(30)$ & \\
\hline$\%$ Stayed negative $(\mathrm{n})$ & $24.1 \%(20)$ & $25.0 \%(8)$ & $23.5 \%(12)$ & 0.866 \\
\hline \% Became positive (n) & $19.3 \%(16)$ & $21.9 \%(7)$ & $17.7 \%(9)$ & \\
\hline$\% \mathrm{BV}$ at CAP002 enrollment $\dagger(\mathrm{n})$ & $55.6 \%(45 / 81)$ & $62.5 \%(20)$ & $51.0 \%(25 / 49)$ & 0.365 \\
\hline$\%$ Trichomonas vaginalis at CAP002 enrollment $\dagger$ (n) & $4.9 \%(4 / 81)$ & $9.4 \%(3)$ & $2.0 \%(1 / 49)$ & 0.295 \\
\hline$\%$ Neisseria gonorrhoeae at CAP002 enrollment $\dagger$ (n) & $6.2 \%(5 / 81)$ & $3.1 \%(1)$ & $8.2 \%(4 / 49)$ & 0.643 \\
\hline$\%$ Chlamydia trachomatis at CAP002 enrollment $\dagger$ (n) & $16.1 \%(13 / 81)$ & $18.8 \%(6)$ & $14.3 \%(7 / 49)$ & 0.758 \\
\hline$\%$ Mycoplasma genitalium at CAP002 enrollment $\dagger(\mathrm{n})$ & $8.6 \%(7 / 81)$ & $12.5 \%(4)$ & $6.1 \%(3 / 49)$ & 0.426 \\
\hline$\%$ HSV-2 PCR at CAP002 enrollment $\dagger$ (n) & $11.1 \%(9 / 81)$ & $12.5 \%(4)$ & $10.2 \%(5 / 49)$ & 0.734 \\
\hline $\begin{array}{l}\% \text { with any STI } \ddagger \text { at CAP002 enrollment excluding } \\
\text { BV (n) }\end{array}$ & $40.7 \%(33 / 81)$ & $43.8 \%(14)$ & $38.8 \%(19 / 49)$ & 0.817 \\
\hline
\end{tabular}

* $P$-value calculated using Wilcoxon rank sums test (medians) or Fisher exact tests (proportions).

$\dagger$ Missing STI data at CAPRISA 002 enrollment for 2 participants from the placebo arm.

\$Any STI defined as having either T. vaginalis, N. gonorrhoeae, C. trachomatis, M. genitalium, HSV-2 PCR, or syphilis.

$\mathrm{BV}$, bacterial vaginosis.

$\mathrm{B} * 27, \mathrm{~B} * 57, \mathrm{~B} * 5801, \mathrm{~B} * 8101$ ), there was a strong association between assignment to the tenofovir gel arm and postinfection VL (Table 3). However, no association was found between trial arm and CD4 count with a mean CD4 count of 463 cells per microliter $(\mathrm{SE}=46.87)$ in women from the tenofovir arm compared with 514 cells per microliter $(\mathrm{SE}=45.44)$ in the placebo arm $(P=0.290)$ during the follow-up period (see Figure, Supplemental Digital Content, http://links.lww.com/QAI/A574).

The multivariate model (Table 3 ) also revealed a weak negative association between the number of used gel applicators women returned and the postinfection VL [effect estimate $-0.10(\mathrm{SE}=0.05), P=0.058]$ and weak positive associations between the number of sex acts in the previous 30 days $[0.09(\mathrm{SE}=0.05), P=0.079]$, not using a condom at the last sex act $[0.49(\mathrm{SE}=0.28), P=0.078]$ and depot medroxyprogesterone acetate use [0.40 (SE $=$ $0.23), P=0.084$ ]

Because the VL assay changed during the course of follow-up and, therefore, could have differentially affected VL estimates, we retested cryopreserved plasma using the same assay (Taqman version 2.0). The association between study arm and VLs remained significant: 4.40 versus 3.97 log copies per milliliter for tenofovir versus placebo women at 12 months postinfection $(P=0.034)$ (see Table, Supplemental Digital Content, http://links.lww.com/QAI/A574).

An unusually high proportion of women $(12 / 45,26.7 \%)$ from the placebo arm had undetectable VLs $(<400$ copies/ $\mathrm{mL})$ compared with women from the tenofovir arm $(1 / 30$, $3.3 \%)(P=0.011)$. Assessment of stored samples from these women using the more sensitive Taqman version 2.0 assay, with a lower limit of detection of 20 copies per milliliter, 


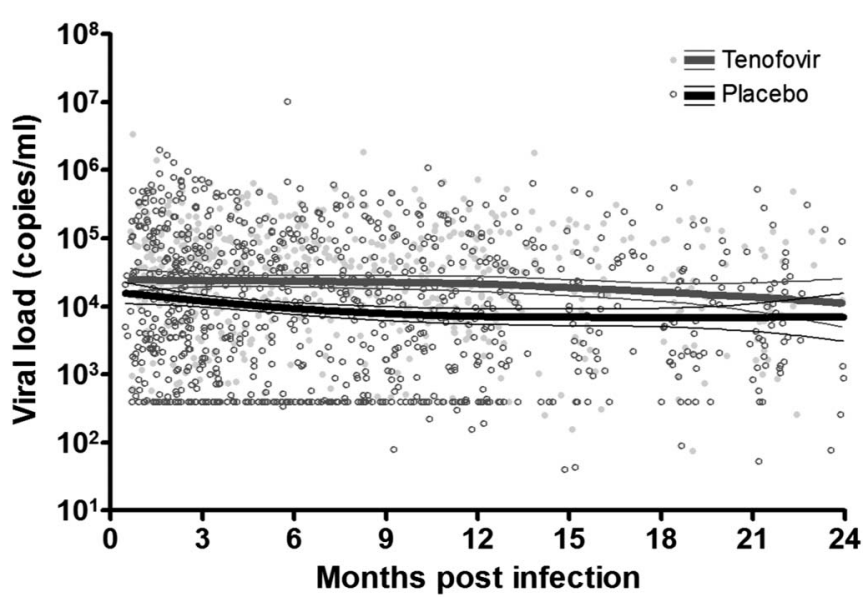

FIGURE 1. Scatterplot of VL measurements within the first 2 years of HIV infection, with a Loess smoothing line and $95 \%$ confidence intervals, stratified by CAPRISA 004 tenofovir gel and placebo arms.

demonstrated that $7(15.6 \%)$ of 45 women versus $1(3.3 \%)$ of 30 women had undetectable VLs $(P=0.134)$ (see Table, Supplemental Digital Content, http://links.lww.com/QAI/A574).

Further analysis of longitudinal data on the 13 women with VL $<400$ copies per milliliter at 12 months have revealed that 2 women have remained virologically suppressed and 3 are viremia controllers (VL $<2000$ copies per milliliter). The remaining women have lost virological control. Antiretroviral drugs (efavirenz, nevirapine, lopinavir, and ritonavir) were undetectable in plasma samples from these 13 women with low VLs, therefore excluding undisclosed ART use.

\section{Genital Tract Tenofovir Levels at Time of Seroconversion are not Associated With VL}

Among women from the tenofovir gel arm of the CAPRISA 004 study, 32 participants had at least 1 tenofovir level measurement from a vaginal aspirate sample measured during preinfection or at first visit postinfection. Mean VL measurements were $4.53(\mathrm{SE}=0.27)$ and $4.60(\mathrm{SE}=0.31) \log$ copies per milliliter in women with detectable versus undetectable tenofovir levels $(P=0.840)$. Participants with detectable and those with undetectable tenofovir levels had a mean CD4 count of 499 cells per microliter $(\mathrm{SE}=87.84)$ and 570 cells per microliter $(\mathrm{SE}=101.06)$, respectively $(P=0.556)$.

\section{Women Who Received Tenofovir and Placebo Gel Have Similar Rates of HIV Disease Progression}

Of the 83 women, $32(38.6 \%)$ reached the main endpoint of 2 consecutive CD4 counts $<350$ cells per microliter, that is, $13(40.6 \%)$ in the tenofovir arm and $19(37.3 \%)$ in the placebo arm (Fig. 2). This happened at a median of 9.4 months (IQR: $5.8-12.5)$ postinfection. There was no association between trial arms and time of reaching CD4 count $<350$ cells per microliter $(P=0.786)$.

\section{DISCUSSION}

Women assigned to tenofovir gel did not have altered HIV disease course, which is an important consideration for topical PrEP rollout. This prospective cohort study found higher HIV VLs among CAPRISA 004 seroconvertors from the tenofovir gel arm compared with the placebo arm.

TABLE 2. Comparison of HIV VL and CD4 Count at 3-Monthly Intervals Postinfection

\begin{tabular}{|c|c|c|c|c|c|c|c|c|}
\hline Month & 3 & 6 & 9 & 12 & 15 & 18 & 21 & 24 \\
\hline \multicolumn{9}{|l|}{ VL (log copies/mL) } \\
\hline \multicolumn{9}{|l|}{ Tenofovir } \\
\hline $\mathrm{n}$ & 28 & 31 & 30 & 30 & 29 & 26 & 26 & 22 \\
\hline Median (IQR) & $\begin{array}{c}4.42 \\
(3.74-4.91)\end{array}$ & $\begin{array}{c}4.69 \\
(3.84-4.99)\end{array}$ & $\begin{array}{c}4.46 \\
(3.98-4.89)\end{array}$ & $\begin{array}{c}4.24 \\
(3.75-4.77)\end{array}$ & $\begin{array}{c}4.23 \\
(3.55-4.81)\end{array}$ & $\begin{array}{c}4.21 \\
(3.49-4.88)\end{array}$ & $\begin{array}{c}3.96 \\
(3.51-4.62)\end{array}$ & $\begin{array}{c}4.10 \\
(3.46-4.86)\end{array}$ \\
\hline \multicolumn{9}{|l|}{ Placebo } \\
\hline $\mathrm{n}$ & 45 & 47 & 46 & 46 & 43 & 37 & 41 & 33 \\
\hline Median (IQR) & $\begin{array}{c}4.03 \\
(3.08-4.84)\end{array}$ & $\begin{array}{c}4.12 \\
(3.13-4.95)\end{array}$ & $\begin{array}{c}4.08 \\
(2.92-4.66)\end{array}$ & $\begin{array}{c}3.70 \\
(2.60-4.66)\end{array}$ & $\begin{array}{c}3.88 \\
(3.23-4.56)\end{array}$ & $\begin{array}{l}3.69 \\
(3.19-4.55)\end{array}$ & $\begin{array}{c}3.76 \\
(3.18-4.44)\end{array}$ & $\begin{array}{l}3.55 \\
(3.12-4.58)\end{array}$ \\
\hline$P^{*}$ & 0.154 & 0.065 & 0.055 & 0.016 & 0.189 & 0.122 & 0.351 & 0.189 \\
\hline \multicolumn{9}{|l|}{ CD4 count (cells $/ \mu \mathrm{L})$} \\
\hline \multicolumn{9}{|l|}{ Tenofovir } \\
\hline $\mathrm{n}$ & 28 & 30 & 30 & 30 & 29 & 26 & 26 & 22 \\
\hline Median (IQR) & $\begin{array}{c}492 \\
(391-631)\end{array}$ & $\begin{array}{c}471 \\
(365-602)\end{array}$ & $\begin{array}{c}443 \\
(393-606)\end{array}$ & $\begin{array}{c}442 \\
(301-578)\end{array}$ & $\begin{array}{c}434 \\
(325-531)\end{array}$ & $\begin{array}{c}476 \\
(367-679)\end{array}$ & $\begin{array}{c}477 \\
(396-592)\end{array}$ & $\begin{array}{c}454 \\
(412-640)\end{array}$ \\
\hline \multicolumn{9}{|l|}{ Placebo } \\
\hline $\mathrm{n}$ & 45 & 47 & 46 & 46 & 43 & 39 & 42 & 34 \\
\hline Median (IQR) & $\begin{array}{c}537 \\
(433-683)\end{array}$ & $\begin{array}{c}487 \\
(374-737)\end{array}$ & $\begin{array}{c}461 \\
(365-672)\end{array}$ & $\begin{array}{c}432 \\
(368-655)\end{array}$ & $\begin{array}{c}480 \\
(352-641)\end{array}$ & $\begin{array}{c}513 \\
(409-653)\end{array}$ & $\begin{array}{c}536 \\
(392-591)\end{array}$ & $\begin{array}{c}510 \\
(374-626)\end{array}$ \\
\hline$P^{*}$ & 0.475 & 0.487 & 0.629 & 0.323 & 0.181 & 0.512 & 0.331 & 1.000 \\
\hline
\end{tabular}

*Wilcoxon rank sums test. 
TABLE 3. Univariate and Multivariate Linear Mixed Model Fitted to HIV VL Within the First 24 Months of Infection

\begin{tabular}{|c|c|c|c|c|}
\hline \multirow[b]{2}{*}{ Variable } & \multicolumn{2}{|c|}{ Univariate Analysis* } & \multicolumn{2}{|c|}{ Multivariate Analysis } \\
\hline & Effect Estimate (SE) & $\boldsymbol{P}$ & Effect Estimate (SE) & $\boldsymbol{P}$ \\
\hline Tenofovir vs placebo & $0.38(0.20)$ & 0.059 & $0.50(0.20)$ & 0.013 \\
\hline Age (years) & $-0.02(0.02)$ & 0.373 & $-0.02(0.03)$ & 0.438 \\
\hline DMPA vs no DMPA $\dagger$ & $0.16(0.20)$ & 0.423 & $0.40(0.23)$ & 0.084 \\
\hline Condom used at last sex act $\dagger$ (no vs yes) & $0.42(0.27)$ & 0.127 & $0.49(0.28)$ & 0.078 \\
\hline Number of sex acts in the past 30 days $\dagger$ & $0.01(0.02)$ & 0.578 & $0.09(0.05)$ & 0.079 \\
\hline Median gel adherence in 004 & $-0.003(0.004)$ & 0.4436 & $0.003(0.005)$ & 0.636 \\
\hline $\begin{array}{l}\text { STI symptoms within } 3 \text { months before } \\
\text { seroconversion (yes vs. no) }\end{array}$ & $-0.12(0.21)$ & 0.562 & $-0.22(0.23)$ & 0.333 \\
\hline \multicolumn{5}{|l|}{ HSV2 status in 004} \\
\hline Baseline positive vs remained negative & $-0.04(0.24)$ & 0.857 & $-0.002(0.26)$ & 0.995 \\
\hline Protective HLA-B type $§$ (yes vs no) & $-0.47(0.22)$ & 0.036 & $-0.35(0.23)$ & 0.141 \\
\hline
\end{tabular}

*All univariate analyses were adjusted for time postinfection and VL assay used (Taqman1 vs Amplicor).

$\dagger$ As reported at last visit before HIV infection in 004.

$\$$ Any STI is defined as having Trichomonas vaginalis, Neisseria gonorrhoeae, Chlamydia trachomatis, Mycoplasma genitalium, HSV-2 PCR, or syphilis

$\S$ Protective HLA-B types defined as B*1302, B*27, B*57, B*5801, B*8101.

DMPA, depot medroxyprogesterone acetate.

However, there was no corresponding difference in CD4 counts or earlier need for ART initiation comparing these 2 groups of seroconvertors. The difference in VL was only partially explained by a higher proportion of women with protective HLA alleles among women from the placebo arm. In addition, the study did not find a dose-response effect when comparing the presence of genital tract tenofovir with VL or CD4 counts. Therefore, although tenofovir gel prophylaxis may be associated with higher VLs, this does not translate into differences in HIV disease course.

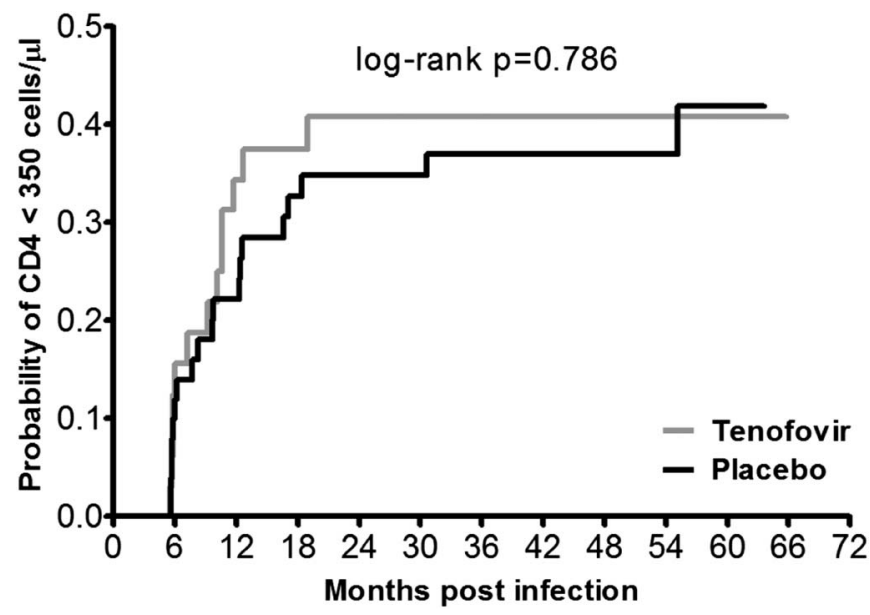

FIGURE 2. Kaplan-Meier graph of time to CD4 count $<350$ cells per microliter stratified by CAPRISA 004 arm.
This is one of the first human studies reporting on longterm follow-up of individuals who seroconverted after exposure to PrEP. Markers of disease progression and possible confounding characteristics were systematically and prospectively collected and retention rates in the study remain high.

The main limitation of this study is possible selection bias. The CAPRISA 004 trial showed a 39\% effectiveness of the $1 \%$ tenofovir gel, thereby protecting some women participating in the tenofovir arm from HIV infection. Once HIV was diagnosed, women were no longer randomized and important population differences may have been selected, as evidenced, for example, by some differences in HLA alleles between women who seroconverted in the 2 arms. Despite this limitation, comparison of baseline demographic, behavioral, and clinical characteristics revealed no obvious differences; thus, any differences must have been subtle or not studied. One of these differences may have been the level of genital tract inflammation at the time of transmission, which was measured to be significantly higher in tenofovir arm seroconvertors, and is most likely one of the main reasons for tenofovir gel failure in well-adherent women. ${ }^{20}$

A recent study by Chirwa et $\mathrm{al}^{14}$ reported on follow-up of 33 seroconvertors ( 9 assigned to tenofovir/emtricitabine and 24 to placebo) from the TDF2 oral PrEP trial. This smaller study found no significant differences in the CD4 count or VL profiles after 2 years of follow-up. Although, to our knowledge, there are no other studies in humans, several macaque monkey studies have previously looked at the period immediately after SHIV acquisition in oral PrEP exposed animals. 
Zheng et $\mathrm{al}^{21}$ found that PrEP was limiting early virus evolution most likely because of the direct antiviral effect of PrEP and/or reduced target cell availability. Kersh et al $^{12}$ reported a 100-fold lower peak viremia and reduced systemic inflammation in their macaque model after oral tenofovir/emtricitabine PrEP exposure. Furthermore, PrEP-treated macaques showed no significant CD4 cell count reduction during acute infection and developed more SHIV-specific central memory $\mathrm{T}$ cells relative to control macaques.

Similar to Kersh et al, ${ }^{12}$ a study by Curtis et $\mathrm{al}^{22}$ found a lower peak viremia during acute infection in tenofovir/ emtricitabine-exposed macaques. Interestingly, although the timing of seroconversion, SHIV binding, and neutralizing antibody levels were not impacted by treatment, lower maturation rates of antibody avidity for anti-p27, gp120, gp160, and gp41 were observed. Whether this could have led to higher VLs later on during the course of SHIV infection is not known. Furthermore, it is important to remember that these studies were all conducted with oral PrEP and in animals. Whether these findings also hold true in humans exposed to a $1 \%$ tenofovir gel microbicide is currently unknown.

Potential mechanisms for an increased VL after tenofovir gel failure could include a selection of fitter viruses at HIV acquisition, although preliminary studies do not support this, higher genital and systemic genital tract inflammation driving higher VLs, or a delay in VL decline after acute infection. To unravel whether the difference in VL in this study is a direct effect of PrEP or because of the selection of 2 distinct cohorts after tenofovir gel exposure, further studies are underway. We have already established that the diversity, fitness, and resistance pattern of the transmitted virus, as well as T-cell responses ${ }^{16-19}$ were not adversely affected by $1 \%$ tenofovir gel exposure and are currently investigating whether a delay in the antibody response of women who used the tenofovir gel could have been a contributing mechanism to explain the difference in VLs.

At the same time, long-term clinical follow-up of participants in this study will continue as part of the CAPRISA 002 study, and in the CAPRISA 009 study, an open-label randomized controlled trial (clinicaltrials.gov ref \# NCT01387022) to assess the impact of prophylactic exposure to tenofovir gel on the efficacy of subsequent tenofovircontaining ART on viral suppression.

\section{ACKNOWLEDGMENTS}

The authors thank all the CAPRISA 002 Acute Infection Study participants who are continuing to make an important personal contribution to HIV research through their support and participation in our studies. The scientific and supportive role of the whole CAPRISA 004 and CAPRISA 002 study and protocol teams are gratefully acknowledged. The authors also thank Jennifer Norman for facilitating the antiretroviral drug level testing in the Division of Clinical Pharmacology, University of Cape Town, South Africa.

\section{REFERENCES}

1. Anderson PL, Glidden DV, Liu A, et al. Emtricitabine-tenofovir concentrations and pre-exposure prophylaxis efficacy in men who have sex with men. Sci Transl Med. 2012;4:151ra125.
2. Choopanya K, Martin M, Suntharasamai P, et al. Antiretroviral prophylaxis for HIV infection in injecting drug users in Bangkok, Thailand (the Bangkok Tenofovir Study): a randomised, double-blind, placebocontrolled phase 3 trial. Lancet. 2013;381:2083-2090.

3. Baeten JM, Donnell D, Ndase P, et al. Antiretroviral prophylaxis for HIV prevention in heterosexual men and women. $N$ Engl J Med. 2012;367: 399-410.

4. Thigpen MC, Kebaabetswe PM, Paxton LA, et al. Antiretroviral preexposure prophylaxis for heterosexual HIV transmission in Botswana. N Engl J Med. 2012;367:423-434.

5. Abdool Karim Q, Abdool Karim SS, Frohlich JA, et al. Effectiveness and safety of tenofovir gel, an antiretroviral microbicide, for the prevention of HIV infection in women. Science. 2010;329:1168-1174.

6. Marrazzo JM, Ramjee G, Nair G, et al; for the VOICE Team. Pre-exposure prophylaxis for HIV in women: daily oral tenofovir, oral tenofoviremtricitabine, or vaginal tenofovir gel in the VOICE Study (MTN 003. Conference for Retrovirusus and Opportunistic Infections, Atlanta, GA; March 4, 2013.

7. McCormack S, Fidler S, Fisher M. The British HIV Association/British Association for Sexual Health and HIV Position Statement on preexposure prophylaxis in the UK. Int J STD AIDS. 2012;23:1-4.

8. Cohen MS, Muessig KE, Smith MK, et al. Antiviral agents and HIV prevention: controversies, conflicts, and consensus. AIDS. 2012;26:1585-1598

9. Peterson L, Taylor D, Roddy R, et al. Tenofovir disoproxil fumarate for prevention of HIV infection in women: a phase 2, double-blind, randomized, placebo-controlled trial. PLoS Clin Trials. 2007;2:e27.

10. Grohskopf LA, Chillag KL, Gvetadze R, et al. Randomized trial of clinical safety of daily oral tenofovir disoproxil fumarate among HIVuninfected men who have sex with men in the United States. $J$ Acquir Immune Defic Syndr. 2013;64:79-86.

11. Liu AY, Vittinghoff E, Chillag K, et al. Sexual risk behavior among HIV-uninfected men who have sex with men participating in a tenofovir preexposure prophylaxis randomized trial in the United States. $J$ Acquir Immune Defic Syndr. 2013;64:87-94.

12. Kersh EN, Luo W, Zheng Q, et al. Reduced inflammation and CD4 loss in acute SHIV infection during oral pre-exposure prophylaxis. $J$ Infect Dis. 2012;206:770-779.

13. Garcia-Lerma JG, Otten RA, Qari SH, et al. Prevention of rectal SHIV transmission in macaques by daily or intermittent prophylaxis with emtricitabine and tenofovir. PLoS Med. 2008;5:e28.

14. Chirwa LI, Johnson JA, Niska RW, et al. CD4+ cell count, viral load, and drug resistance patterns among heterosexual breakthrough HIV infections in a study of oral preexposure prophylaxis. AIDS. 2014;28:223-226.

15. van Loggerenberg F, Mlisana K, Williamson C, et al. Establishing a cohort at high risk of HIV infection in South Africa: challenges and experiences of the CAPRISA 002 acute infection study. PLoS One. 2008;3:e1954.

16. Valley-Omar Z, Sibeko S, Anderson J, et al. CAPRISA 004 tenofovir microbicide trial: no impact of tenofovir gel on the HIV transmission bottleneck. J Infect Dis. 2012;206:35-40.

17. Chopera DR, Mann JK, Mwimanzi P, et al. No evidence for selection of HIV1 with enhanced gag-protease or Nef function among breakthrough infections in the CAPRISA 004 tenofovir microbicide trial. PLoS One. 2013;8:e71758.

18. Mureithi MW, Poole D, Naranbhai V, et al. Preservation HIV-1-specific IFNgamma + CD4+ T-cell responses in breakthrough infections after exposure to tenofovir gel in the CAPRISA 004 microbicide trial. $J$ Acquir Immune Defic Syndr. 2012;60:124-127.

19. Wei X, Hunt G, Abdool Karim SS, et al. Sensitive tenofovir resistance screening of HIV-1 from the genital and blood compartments of women with breakthrough infections in the CAPRISA 004 tenofovir gel trial J Infect Dis. 2014;209:1916-1920.

20. Roberts LP, Passmore J, Williamson C, et al. Genital tract inflammation in women participating in the CAP tenofovir microbicide trial who became infected with HIV: a mechanism for breakthrough infection? Conference for Retrovirusus and Opportunistic Infections, Boston, MA; March 3, 2011.

21. Zheng Q, Ruone S, Switzer WM, et al. Limited SHIV env diversification in macaques failing oral antiretroviral pre-exposure prophylaxis. Retrovirology. 2012;9:40.

22. Curtis KA, Kennedy MS, Luckay A, et al. Delayed maturation of antibody avidity but not seroconversion in rhesus macaques infected with simian HIV during oral pre-exposure prophylaxis. J Acquir Immune Defic Syndr. 2011;57:355-362. 